\title{
Extreme conservation of noncoding DNA near HoxD complex of vertebrates
}

\author{
Chilaka Sabarinadh, Subbaya Subramanian, Anshuman Tripathi and \\ Rakesh K Mishra*
}

Address: Centre for Cellular and Molecular Biology, Uppal Road, Hyderabad 500 007, India

Email: Chilaka Sabarinadh - sabarinadhch@yahoo.com; Subbaya Subramanian - subree@stanford.edu; Anshuman Tripathi - anshu411@rediffmail.com; Rakesh K Mishra*-mishra@ccmb.res.in

* Corresponding author

Published: 06 October 2004

BMC Genomics 2004, 5:75 doi:10.1 I86/I47|-2/64-5-75
Received: 23 April 2004

Accepted: 06 October 2004

This article is available from: http://www.biomedcentral.com/I47I-2/64/5/75

(c) 2004 Sabarinadh et al; licensee BioMed Central Ltd.

This is an open-access article distributed under the terms of the Creative Commons Attribution License (http://creativecommons.org/licenses/by/2.0), which permits unrestricted use, distribution, and reproduction in any medium, provided the original work is properly cited.

\begin{abstract}
Background: Homeotic gene complexes determine the anterior-posterior body axis in animals. The expression pattern and function of hox genes along this axis is colinear with the order in which they are organized in the complex. This 'chromosomal organization and functional correspondence' is conserved in all bilaterians investigated. Genomic sequences covering the HoxD complex from several vertebrate species are now available. This offers a comparative genomics approach to identify conserved regions linked to this complex. Although the molecular basis of 'colinearity' of Hox complexes is not yet understood, it is possible that there are control elements within or in the proximity of these complexes that establish and maintain the expression patterns of hox genes in a coordinated fashion.
\end{abstract}

Results: We have compared DNA sequence flanking the HoxD complex of several primate, rodent and fish species. This analysis revealed an unprecedented conservation of non-coding DNA sequences adjacent to the HoxD complex from fish to human. Stretches of hundreds of base pairs in a $7 \mathrm{~kb}$ region, upstream of HoxD complex, show $100 \%$ conservation across the vertebrate species. Using PCR primers from the human sequence, these conserved regions could be amplified from other vertebrate species, including other mammals, birds, reptiles, amphibians and fish. Our analysis of these sequences also indicates that starting from the conserved core regions, more sequences have been added on and maintained during evolution from fish to human.

Conclusion: Such a high degree of conservation in the core regions of this $7 \mathrm{~kb}$ DNA, where no variation occurred during $\sim 500$ million years of evolution, suggests critical function for these sequences. We suggest that such sequences are likely to provide molecular handle to gain insight into the evolution and mechanism of regulation of associated gene complexes.

\section{Background}

Eukaryotic genome contains a large excess of non-coding sequences. Conservation of these sequences among species is a strong indication of their functional significance.
With the availability of genome sequences it is possible to identify such sequences taking a comparative genomics approach [1-4]. The clusters of homeotic genes, which are expressed in a coordinated manner [5], are among the 


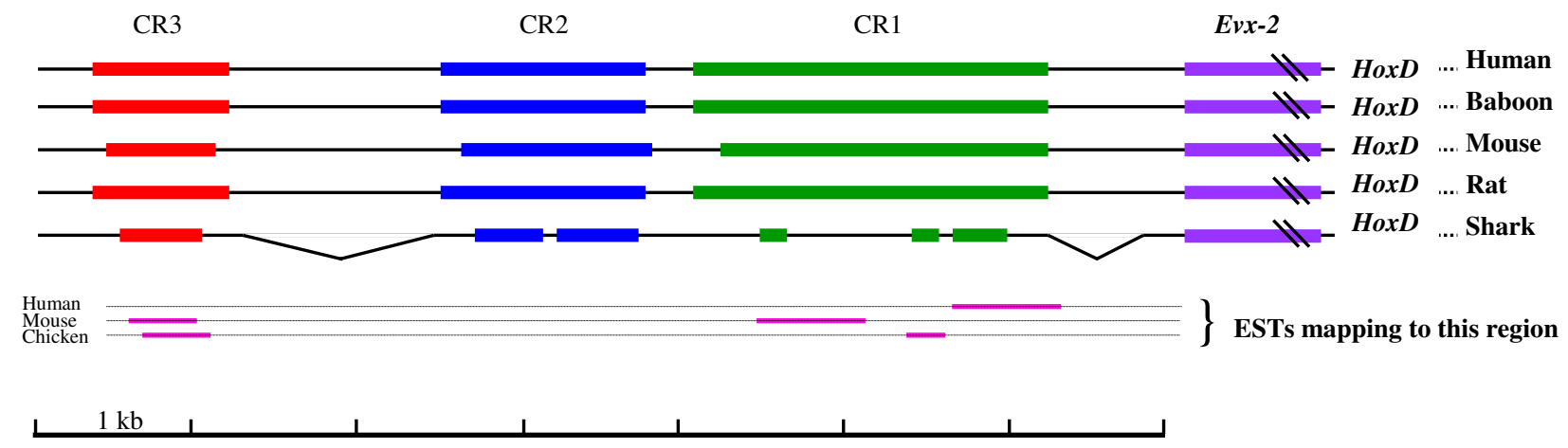

Figure I

Schematic representation of sequence conservation in the HoxD upstream region. Human sequence (AC009336; from position 5660I to 64095) was compared to the corresponding sequences of Papio hamadryas (ACI I6665), Heterodontus francisci (AF224263), Mus musculus (AC0I5584), Fugu rubripes (CAABOI000449) and Rattus norvegicus (NW_042732). Sequences that are conserved across vertebrates are shown as blocks. The conservation extends beyond these blocks within primates and rodents. ESTs found in the database corresponding to this region are also shown. ESTs mapping to CR3 are BB838602 from mouse 8 cell embryo and BUI29I54 from chicken 36 stage limb; and those mapping to CRI are AA620964 from human testis; BB332383, BB335 I IO, BB334358, BB333569 from 6 and I 0 days mouse neonate medulla oblongata and BU255316 from chicken 36 stage limb.

most conserved regions of the vertebrate genome. Clustering of genes that are regulated in a linked manner has been noticed in several other cases $[6,7]$. However, the molecular mechanism behind such coordination in regulation is not yet understood. Several mechanisms have been proposed that link the organization of homeotic genes and the spatio-temporally controlled expression [8]. Colinearity in hox complexes was first discovered in Drosophila [9] and later studies on the bithorax complex have demonstrated the role of chromatin organization in its regulation [10]. Recent studies on the HoxD complex suggest a role for higher order chromatin organization in the regulation of this complex involving up to $20 \mathrm{~kb}$ upstream region [11].

\section{Results and discussion}

We compared genomic regions flanking hox complexes in order to identify conserved regions with potential regulatory function. Here we report that the upstream regions of HoxD complexes of human, mouse, rat, sacred baboon, horn shark, zebra fish and puffer fish contain long stretches of extremely conserved sequences. In the $25 \mathrm{~kb}$ region upstream of the HoxD complex from these organisms we found an extremely conserved region spread in three blocks located within $7 \mathrm{~kb}$ from the 3 ' end of the Evx-2 gene. These conserved regions, designated as Conserved Region 1 , Conserved Region $\underline{2}$ and Conserved Region $\underline{3}$ (CR1, CR2 and CR3) (Fig. 1) show a degree of conservation not seen before among distant species.
Detailed analysis of each region spanning to several hundred base pairs, in particular the CR2 shows several stretches of $100 \%$ conservation, Fig. 2. We also noticed longer stretches of conservation among mammals, which gradually shortens as we go towards lower vertebrates, defining the core of each conserved region, across the vertebrate classes, see Additional file 1 . This and the fact that in case of shark, as compared to mammals, the intervening sequence lengths between CR2 and CR3, and CR1 and Evx-2 is shorter by $\sim 1300$ bp and $\sim 600 \mathrm{bp}$, respectively (Fig. 1) suggest that starting from the shorter conserved regions, additional unique sequences have progressively been acquired and conserved during the evolution of primates from lower vertebrates. This may reflect the molecular basis of conservation and elaboration of Hox gene regulation during evolution of these species [12].

Universal occurrence of these sequences in all vertebrate classes was confirmed by their amplification using primers from human HoxD complex (Figure 3) followed by Southern hybridization and sequencing (unpublished observation). Furthermore, using CR1, CR2 or CR3 as query we searched genomic sequences of variety of eukaryotes in available databases. This search indicated that these sequences are single copy and vertebrate specific. While these conserved regions appear to be a key component of the HoxD complex of all vertebrates looked at, we did not find such a degree of conservation in the flanking regions of other hox complexes (HoxA, $B$ and $C$ ) 


\section{CR3}

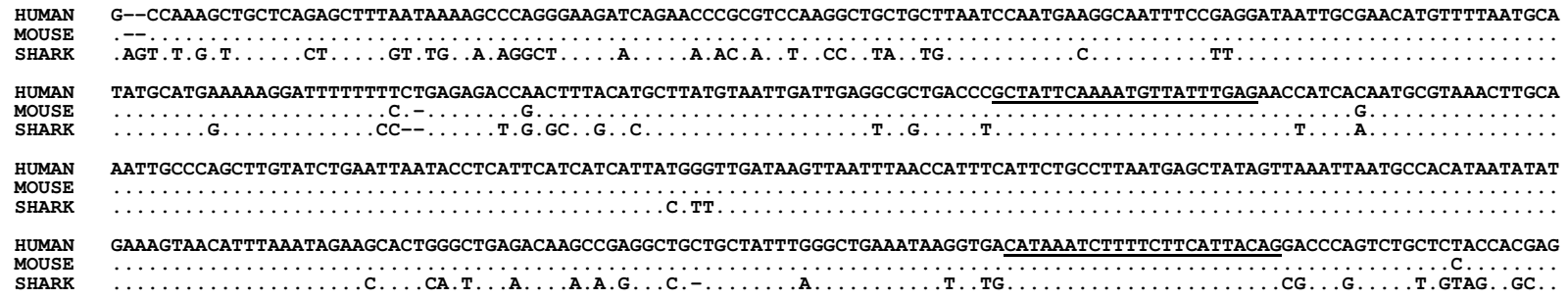

CR2

HUMAN
MOUSE
SHARK
HUMAN
MOUSE
SHARK
HUMAN
MOUSE
SHARK
HUMAN
MOUSE
SHARK
HUMAN
MOUSE
SHARK
HUMAN
MOUSE
SHARK
HUMAN
MOUSE
SHARK
HUMAN
MOUSE
SHARK
CRR1
COUMAN
MOUSE
SHARK
HUMAN
MOUSE
SHARK
HUMAN
MOUSE
SHARK
HUMAN
MOUSE
SHARK

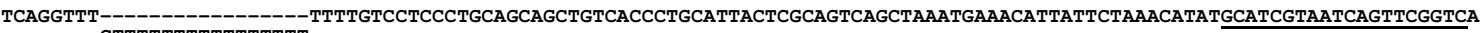

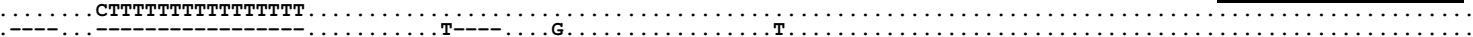
CACTTACAAGAACACGCGTTAATAAGGCAATCAATCACCCTGGAACAAGCAAGTTGTTCTGTAACAGCTCATAAACAGTGTGTAATGAAGAATTGGAGGTTACCGTGACATGCGTTGATC

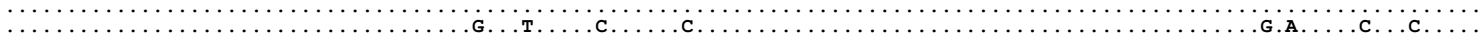
AGATAATCAATGTCAAAGATGCGATGAATGTCAGTAAATGTAGTTTTCATGTCGTTTCTATAAAATCTTCAATTTACAACAAGCAGTTCAATTACCCAGAAAATACAGTCAATTAAATAG

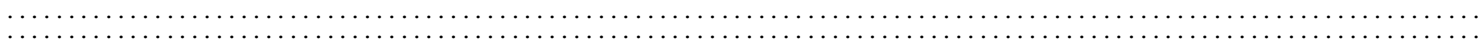
GGGTGATTGGACAGTAGGGGGTGGATCATCGATCTTTGCATTTCTATCTCGCTAGTGGACATTTAATTTGGTTTTTCCATTAGCGACGAAATAAAGAAAATATAAAATATATTAAACAA

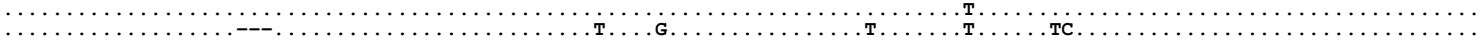
ССTACAGATTTATTTTCTTGTCAAAAACAATTCGGGCTTGATGACAGACAGTCTTCTGCATTTTATGATGAATTATTTTTTCATTCTTTGCACACTCGAGACAAAAAAAA-----------… ----тGCTGTTATTTTGGACAGGGTTTTTTGGCCA-----------CTGTCTTTTTCCGTTTGCTGGCCCATCTATCTCAATGCTTTTGTTTTTAAGGTTACAACCCCCGAGTTAGCA

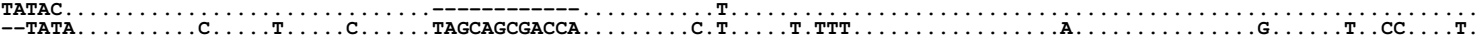
AAGAGCACCGAAAACCAGGGTGATACATCACCAGTCCAAATGTGCTGCTATAATCGTATTTCTTTAATGGGGGAGTTCAAGAAAAGAGAGAAGGGGAAAGAGAGAAAAGAATTTATC----

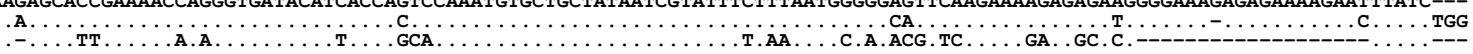
GAGGGGGAGGGAACCCTTGGGATTACTTCAGTATATATTTAACCAACCTGCAATTAAAGACGGTATCAGCTTGTATCATTTAGAGCTAATGCAATAATAATGGTAAATTACAGGCC-AAA AG.

CR1

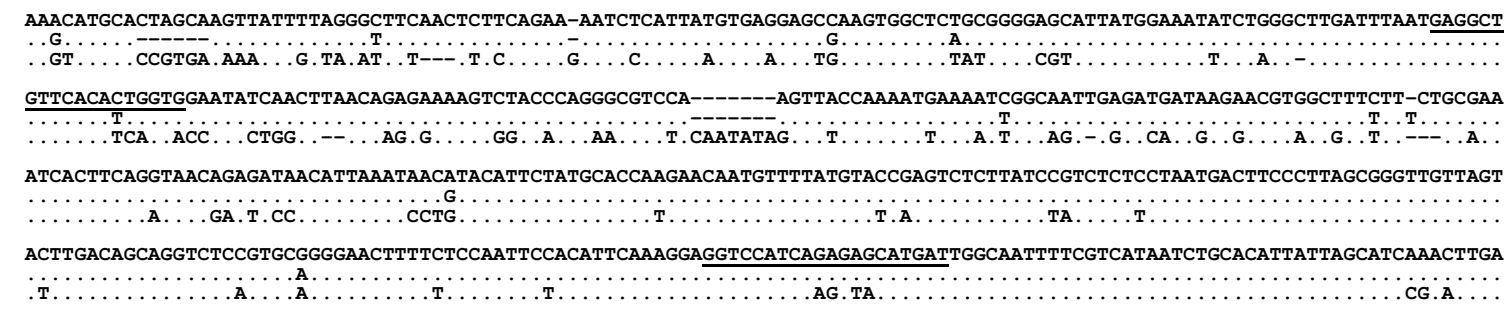

Figure 2

Comparison of conserved regions from human, mouse and shark. Conserved bases of mouse and shark are shown as '.' and '-' indicates indels. Underlined sequences of human indicate primers that were used for amplification of the corresponding sequence from different vertebrates.

of vertebrates. In order to trace back the evolutionary origin of such sequences, it will be of interest to investigate occurrence of these sequences at the corresponding region in the hox complexes of species of urochordata, cephalochordata or even agnatha. In the tunicate Oikopleura dioca, where hox genes are dispersed but the spatial pattern seen in other animals is still present [13], we did not find CR1, CR2 or CR3. Also, we did not find any significant conserved region corresponding to these CRs in the amphioxus genomic region that contains the cluster of hox genes. It appears, therefore, that these extremely conserved sequences have originated in the vertebrates where the hox complex has additional distinct features of tight clustering compared to the insect hox clusters and the temporal colinearity, not seen in invertebrates.

Several recent reports using comparative genomics approach have identified conserved non-coding regions 


\section{M hu mo ch co fr zf}

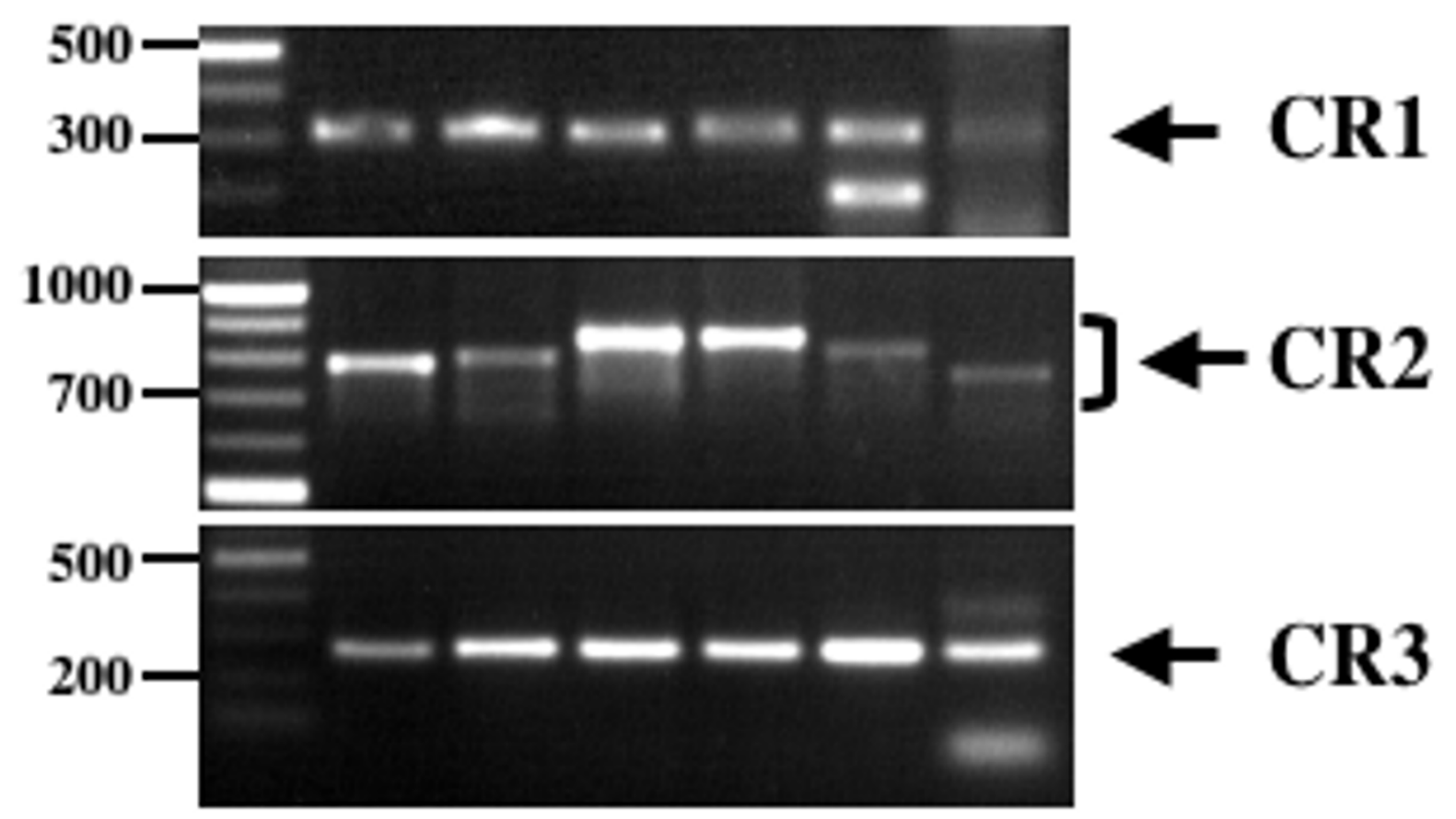

Figure 3

Conservation of CRI, CR2 and CR3 in all vertebrate classes. PCR amplification of different vertebrate genomic DNA samples using primers designed based on the human sequence. Lanes: $M$ - size marker indicated in bp, hu - human, mo mouse, ch - chicken, co - cobra, fr - frog and $\mathrm{zf}$ - zebra fish. The arrows indicate the corresponding products that have been confirmed by direct sequencing as well as Southern hybridization using human CRs as probe.

among different vertebrates [14-16] but none to the degree that we report here. The mechanism that may require such a high degree of conservation is not known. It is not, therefore, immediately clear what precisely is the role of these sequences. EST database search revealed that part of CR1 and CR3 are transcribed without any significant ORF but no EST corresponding to CR2 or any other part of the $7 \mathrm{~Kb}$ region was found, Fig. 1. A possible mechanism could involve RNA from this region that may function by base pairing to the genomic target sites. If that is the case, such high conservation could be expected. Role of transcription in the regulation of bithorax complex is emerging from recent studies [17].

\section{Conclusions}

While such an extreme conservation of several hundred nucleotides over half a billion years in a region that does not code for any known proteins certainly implicates essential role for such sequences, probably in the regulation of HoxD complex, no known regulatory element requires such extreme conservation extending up to hundreds of base pairs. It is, therefore, likely that these elements could be components of a novel mechanism common to all vertebrates that regulates this gene complex. We are tempted to suggest that such a strongly conserved region from fish to human linked to a gene complex that is known to determine body axis formation may be the key determinant of molecular basis of early ontogeny. Early embryos of all vertebrates show striking similarity and we suggest that these elements may control the early expression pattern of HoxD which leads to similar pattern of the embryo shape. The gradient of conservation seen in this region from fish to human may further signify the evolutionary history of this locus and 
diversification of the morphological features along the anterior-posterior body axis of the vertebrate classes.

\section{Methods}

\section{Sequence analysis}

The genomic sequences that contained Evx-2 and any of the Hoxd genes were downloaded and annotated using gene/ORF prediction tools. Similar approach was used for other hox complexes. Homology searches of the upstream sequences of HoxD region from human (AC009336; from nucleotide 56601 to 64095) was carried out using the BLAST program of NCBI. The sequences that showed significant homology were further used to analyze the extent of homology by BLAST 2 program. The conserved regions from each sequence was obtained and subjected to multiple sequence analysis using Clustal X. In order to identify the expressed sequences corresponding to the conserved sequence, the conserved sequences along with the unique sequences were BLASTed against EST databases (human, mouse and dbEST).

The contigs that showed significant homology to the upstream sequences of human HoxD were annotated using the tBLASTx program and searching the translated amino acid sequence in the Swissprot database. Repeat masker program was used to look for repeat content. Genebank sequences used in this study are as follows: AC1 16665 Papio hamadryas, AF224263 Heterodontus francisci, AC015584 Mus musculus, AC009336 Homo sapiens, CAAB01000449 Fugu rubripes and NW_042732 Rattus norvegicus.

\section{DNA isolation, PCR amplification, sequencing and Southern hybridization}

For the isolation of genomic DNA blood samples of human, chick and cobra (Naja naja) were used while liver tissue of mouse and muscle tissue of frog (Bufo melanostictus) and zebra fish were used. Standard protocol of DNA isolation was followed which included lysis, RNase A and proteinase $\mathrm{K}$ digestions followed by phenol/chloroform extraction and precipitation. Concentration and quality of the genomic DNA was checked on $0.7 \%$ agarose gel and UV absorption spectrophotometry. Based on the sequence of conserved regions primers were designed to amplify the three regions CR1, CR2 and CR3.

Primers used in this study to amplify conserved regions from different vertebrate species were:CR1 forward- GAGGCTGTTCACACTGGTGG,CR1 reverseATCATGCTCTCTGATGGACC,CR2 forward- GCATCGTAATCAGTTCGGTC,CR2 reverse- TGATACAAGCTGATACCGTC,CR3 forward- GCTATTCAAAATGTTATTTGAG and CR3 reverse- CTGTAATGAAGAAAAGATTTATG.
The $25 \mu \mathrm{l}$ reaction was performed using $100 \mathrm{ng}$ template DNA and 5 pmol each of forward and reverse primers. PCR protocol was as follows: initial denaturation step of $94^{\circ} \mathrm{C}$ for 3 min was followed by 35 cycles of $94^{\circ} \mathrm{C}$ for 1 $\min , 57^{\circ} \mathrm{C}$ for $1 \mathrm{~min}$ and $72^{\circ} \mathrm{C}$ for $1.30 \mathrm{~min}$ and final extension step at $72^{\circ} \mathrm{C}$ for $7 \mathrm{~min}$. Authenticity of the PCR products was confirmed by direct sequencing and Southern hybridization, using the corresponding human DNA as probe.

\section{Note}

An earlier version of this article was deposited in the 'Deposited Research' section of Genome Biology, http:// genomebiology.com/2003/4/4/P2, [18]. While this manuscript was in reviewing process, a report comparing human genome to several other mammalian sequences identified many highly conserved noncoding sequences [19]. Interestingly, this study also identified CR2 as uc.108 near HOXD and, in agreement to our observation, noted only a "core" conserved region in fish, suggesting that additional parts of the ultraconserved region were innovations after the common ancestor with fish.

\section{Authors' contributions}

CS carried out the sequence analysis, PCR amplification and Southern analysis. SS participated in sequence analysis and DNA isolation from several organisms. AT carried out the sequencing of PCR products and participated in the sequence alignments. RKM conceived of the study, and participated in its design and coordination. All authors read and approved the final manuscript.

\section{Additional material}

\section{Additional File 1}

Size and degree of conservation of CR1, CR2 and CR3 in different vertebrates. Core of conserved regions and extended conserved regions between indicated species is shown as length of sequence and degree of conservation. Non-overlapping blocks of vertebrate conservation is indicated based on human, baboon, rat, mouse and shark comparison. Click here for file

[http://www.biomedcentral.com/content/supplementary/14712164-5-75-S1.doc]

\section{Acknowledgements}

This work was supported by a young investigators grant (RGY03 I6/200IM) from Human Frontier Science Program to RKM.

\section{References}

I. Pennacchio LA, Rubin EM: Genomic strategies to identify mammalian regulatory sequences. Nature Rev Genet 200I, 2:100-109.

2. Kondrashov AS, Shabalina SA: Classification of common conserved sequences in mammalian intergenic regions. Human Mol Genet 2002, I I:669-674.

3. Dehal P, Predki P, Olsen AS, Kobayashi A, Folta P, Lucas S, Land M, Terry A, Ecale Zhou C, Rash S, Zhang Q, Gordon L, Kim J, Elkin C, 
Pollard MJ, Richardson P, Rokhsar D, Uberbacher E, Hawkins T, Branscomb E, Stubbs L: Human Chromosome 19 and Related Regions in Mouse: Conservative and Lineage-Specific Evolution. Science 200 I, 293: 104- III.

4. Glazko GV, Koonin EV, Rogozin IB, Shabalina SA: A significant fraction of conserved noncoding DNA in human and mouse consists of predicted matrix attachment regions. Trends Genet 2003, 19:119-124.

5. McGinnis W, Krumlauf R: Homeobox genes and axial patterning. Cell 1992, 68:283-302.

6. Boutanaev AM, Kalmykova Al, Shevelyov YY, Nurminsky DI: Large clusters of co-expressed genes in the Drosophila genome. Nature 2002, 420:666-669.

7. Lercher MJ, Urrutia AO, Hurst LD: Clustering of housekeeping genes provides a unified model of gene order in the human genome. Nat Genet 2002, 31:180-183.

8. Duboule D: Vertebrate hox gene regulation: clustering and/or colinearity? Curr Opin Genet Dev 1998, 8:5| 4-5।8.

9. Lewis EB: A gene complex controlling segmentation in Drosophila. Nature 1978, 276:565-570.

10. Mihaly J, Hogga I, Barges S, Galloni M, Mishra RK, Hagstrom K, Muller M, Schedl P, Sipos L, Gausz J, Gyurkovics H, Karch F: Chromatin domain boundaries in the Bithorax complex. Cell Mol Life Sci 1998, 54:60-70.

II. Kondo T, Duboule D: Breaking colinearity in the mouse HoxD complex. Cell 1999, 97:407-4I7.

12. Manzanares M, Wada H, Itasaki N, Trainor PA, Krumlauf R, Holland PW: Conservation and elaboration of Hox gene regulation during evolution of the vertebrates. Nature 2000, 408:854-857.

13. Seo HC, Edvardsen RB, Maeland AD, Bjordal M, Jensen MF, Hansen A, Flaat M, Weissenbach J, Lehrach $H$, Wincker $P$, Reinhardt $R$, Chourrout D: Hox cluster disintegration with persistent anteroposterior order of expression in Oikopleura dioica. Nature 2004, 43I:67-7I.

14. Wassermann WW, Palumbo M, Thompson W, Fickett JW, Lawrence CE: Human-mouse genome comparisons to locate regulatory sites. Nat Genet 2000, 26:225-228.

15. Aparicio S, Morrison A, Gould A, Gilthorpe J, Chaudhuri C, Rigby $P W J$, Krumlauf R, Brenner S: Detecting conserved regulatory elements with the model genome of the Japanese puffer fish Fugu rubripes. Proc Natl Acad Sci USA 1995, 92: I 684-1688.

16. Dermitzakis ET, Reymond A, Lyle R, Scamuffa N, Ucla G, Deutsch S, Stevenson BJ, Flegel V, Bucher P, Jongeneel CV, Antonarakis SE: Numerous potentially functional but non-genic conserved sequences on human chromosome 21. Nature 2002, 420:578-582.

17. Drewell RA, Bae E, Burr J, Lewis EB: Transcription defines the embryonic domains of cis-regulatory activity at the Drosophila bithorax complex. Proc Natl Acad Sci USA 2002, 99:16853-16858.

18. Sabarinadh Ch, Subramanian S, Mishra RK: Extreme conservation of non-repetitive non-coding regions near HoxD complex of vertebrates. Genome Biology 2003, 4:P2.

19. Bejerano G, Pheasant M, Makunin I, Stephen S, Kent WJ, Mattick JS, Haussler D: Ultraconserved elements in the human genome. Science 2004, 304: I 32 I-I 325.

\section{Publish with Biomed Central and every scientist can read your work free of charge}

"BioMed Central will be the most significant development for disseminating the results of biomedical research in our lifetime. "

Sir Paul Nurse, Cancer Research UK

Your research papers will be:

- available free of charge to the entire biomedical community

- peer reviewed and published immediately upon acceptance

- cited in PubMed and archived on PubMed Central

- yours - you keep the copyright
BioMedcentral 